\title{
INVOLUTORY HOPF ALGEBRAS
}

\author{
D. S. PASSMAN AND DECLAN QUINN
}

\begin{abstract}
In 1975, Kaplansky conjectured that a finite-dimensional semisimple Hopf algebra is necessarily involutory. Twelve years later, Larson and Radford proved the conjecture in characterisitic 0 and obtained significant partial results in positive characteristics. The goal of this paper is to offer an efficient proof of these results using rather minimal prerequisites, no "harpoons", and gratifyingly few "hits".
\end{abstract}

Let $H$ be a finite-dimensional Hopf algebra over a field $K$, and let $S: H \rightarrow H$ denote its antipode. Then $H$ is said to be involutory if $S^{2}=\mathrm{id}_{H}$ or equivalently if $S$ is an involution on $H$. In [K], Kaplansky conjectured that a semisimple Hopf algebra is necessarily involutory, and in a fundamental paper [LR2], Larson and Radford proved this assertion at least for fields of characteristic 0 . Furthermore, they showed that if $H$ is semisimple and cosemisimple and if char $K=p>\left(\operatorname{dim}_{K} H\right)^{2}$, then $H$ must again be involutory. A nice exposition of this material can be found in $[\mathrm{N}]$.

While [LR2] is reasonably brief, it depends heavily on certain earlier results on characters of Hopf algebras as contained in [L] and [LR 1], and the combined proof is substantial. The goal of this paper is to present an efficient proof of the main results of [LR2] requiring minimal prerequisites. As will be apparent, our proof follows the same basic outline as that of Larson and Radford, and therefore we offer nothing new in this regard. However, by computing traces on certain tensor modules, we are able to obtain the necessary character formulas quite quickly, and this is where the simplifications occur. In some sense, the hero of this approach is the linear transformation $T$ described below.

If $V$ is a left $H$-module, then $H \otimes V$ is well known to be $H$-isomorphic to $H \otimes V_{\epsilon}$, where $V_{\epsilon}$ is equal to $V$ but with the trivial action determined by the counit $\epsilon$. This is proved quite simply by considering the map $T: H \otimes V \rightarrow H \otimes V$ given by

$$
T: h \otimes v \mapsto \sum_{(h)} h_{1} \otimes h_{2} v \quad \text { for all } h \in H, v \in V .
$$

Indeed, one shows that $T$ is invertible with inverse

$$
T^{-1}: h \otimes v \mapsto \sum_{(h)} h_{1} \otimes S\left(h_{2}\right) v
$$

Received by the editors July 13, 1993 and, in revised form, June 8, 1994; originally communicaated to the Proceedings of the AMS by Lance W. Small.

1991 Mathematics Subject Classification. Primary 16W30.

Research supported in part by NSF Grants DMS-9224662 and DMS-9003374. 
and that $T$ intertwines the two representations. We will not need the latter fact here. Rather, we will just use $T$ and several of its variants to obtain certain trace equations of interest. Specifically, if $\mu: H \otimes V \rightarrow H \otimes V$ is a linear transformation, then $\operatorname{tr}_{H \otimes V} T^{-1} \mu T=\operatorname{tr}_{H \otimes V} \mu$ and, as we will see, equalities of this nature yield the necessary character formulas. We start with a simple example.

Let $\tau: H \rightarrow K$ be the element of the dual $H^{*}$ corresponding to the trace map on $H$. In other words,

$$
\tau(a)=\operatorname{tr}_{H} a_{\mathscr{L}} \quad \text { for all } a \in H
$$

where $a_{\mathscr{L}} \in \operatorname{End}_{K} H$ denotes left multiplication by $a$.

Lemma 1. If $\tau \in H^{*}$ is the trace element described above, then

$$
\tau * \tau=\tau(1) \tau=\left(\operatorname{dim}_{K} H\right) \tau
$$

where $*$ is the convolution multiplication of $H^{*}$. Furthermore, if $s: H^{*} \rightarrow H^{*}$ is the antipode of $H^{*}$, then $s^{2}$ and left multiplication by $\tau$ commute as operators on $H^{*}$.

Proof. Let $V$ be any left $H$-module, and define $T_{0}: H \otimes V \rightarrow H \otimes V$ by

$$
T_{0}: h \otimes v \mapsto \sum_{(h)} h_{1} \otimes S\left(h_{2}\right) v .
$$

Then $T_{0}$ is invertible with inverse given by

$$
T_{0}^{-1}: h \otimes v \mapsto \sum_{(h)} h_{1} \otimes h_{2} v
$$

Now, for any $a \in H$,

$$
\tau(a) \operatorname{dim}_{K} V=\left(\operatorname{tr}_{H} a_{\mathscr{L}}\right) \operatorname{dim}_{K} V=\operatorname{tr}_{H \otimes V}\left(a_{\mathscr{L}} \otimes 1\right)=\operatorname{tr}_{H \otimes V} T_{0}^{-1}\left(a_{\mathscr{L}} \otimes 1\right) T_{0}
$$

and furthermore

$$
T_{0}^{-1}\left(a_{\mathscr{L}} \otimes 1\right) T_{0}: h \otimes v \mapsto \sum_{(a)(h)} a_{1} h_{1} \otimes a_{2} h_{2} S\left(h_{3}\right) v=\sum_{(a)} a_{1} h \otimes a_{2} v
$$

Thus we see that

$$
\operatorname{tr}_{H \otimes V} T_{0}^{-1}\left(a_{\mathscr{L}} \otimes 1\right) T_{0}=\sum_{(a)} \operatorname{tr}_{H}\left(a_{1}\right) \operatorname{L}_{V} \operatorname{tr}_{V} a_{2}=\sum_{(a)} \tau\left(a_{1}\right) \operatorname{tr}_{V} a_{2}
$$

Finally, let $V=H$ so that $\operatorname{dim}_{K} H=\tau(1)$ and $\operatorname{tr}_{V} b=\operatorname{tr}_{H} b_{\mathscr{L}}=\tau(b)$ for all $b \in H$. Then we obtain

$$
\tau(a) \tau(1)=\sum_{(a)} \tau\left(a_{1}\right) \tau\left(a_{2}\right)=(\tau * \tau)(a)
$$

and, since this holds for all $a \in H$, we conclude that $\tau(1) \tau=\tau * \tau$.

For the second part, recall that the antipode $S$ of $H$ is invertible as a linear transformation since $H$ is finite dimensional. Thus, $S^{2}$ is an algebra automorphism of $H$ and this implies that

$$
\tau\left(S^{2}(h)\right)=\operatorname{tr}_{H} S^{2}(h)_{\mathscr{L}}=\operatorname{tr}_{H} h_{\mathcal{L}}=\tau(h)
$$

for all $h \in H$. Furthermore, since $s: H^{*} \rightarrow H^{*}$ is defined by

$$
s(\xi): h \mapsto \xi(S(h)) \quad \text { for all } h \in H, \xi \in H^{*},
$$


the preceding formula translates to the assertion that $s^{2}(\tau)=\tau$. Finally, we use the fact that $s^{2}$ is an algebra automorphism of $H^{*}$ to deduce that

$$
s^{2} \tau_{\mathscr{L}}: \xi \mapsto s^{2}(\tau * \xi)=s^{2}(\tau) * s^{2}(\xi) \quad \text { for all } \xi \in H^{*}
$$

and

$$
\tau_{\mathscr{L}} s^{2}: \xi \mapsto \tau * s^{2}(\xi) \quad \text { for all } \xi \in H^{*} \text {. }
$$

But $s^{2}(\tau)=\tau$, so $s^{2} \tau_{\mathscr{L}}=\tau_{\mathscr{L}} s^{2}$ and the lemma is proved.

It can be shown that $s(\tau)=\tau$, but this is not needed here. On the other hand, we do require the following simple, but extremely useful, observation.

Lemma 2. Let $A$ and $B$ be finite-dimensional $K$-vector spaces, and let $f$ and $f^{\prime}$ be linear transformations from $A$ to $A \otimes\left(\operatorname{End}_{K} B\right)$ with the property that the operators $(\xi \otimes 1) f(a),(\xi \otimes 1) f^{\prime}(a) \in \operatorname{End}_{K} B$ have the same trace for every $a \in A$ and $\xi \in A^{*}=\operatorname{Hom}_{K}(A, K)$. Now let $g: A \otimes B \rightarrow A \otimes B$ be defined by

$$
g: A \otimes B \stackrel{f \otimes 1}{\longrightarrow} A \otimes\left(\operatorname{End}_{K} B\right) \otimes B \stackrel{1 \otimes \mathrm{ev}}{\longrightarrow} A \otimes B
$$

where ev: $\left(\operatorname{End}_{K} B\right) \otimes B \rightarrow B$ is the evaluation map, and let $g^{\prime}: A \otimes B \rightarrow A \otimes B$ be defined similarly using $f^{\prime}$. Then $\operatorname{tr}_{A \otimes B} g=\operatorname{tr}_{A \otimes B} g^{\prime}$.

Proof. Let $\left\{a_{1}, a_{2}, \ldots, a_{m}\right\}$ be a basis for $A$, and for all $i=1,2, \ldots, m$, write

$$
f\left(a_{i}\right)=\sum_{j=1}^{m} a_{j} \otimes f_{i, j} \quad \text { and } \quad f^{\prime}\left(a_{i}\right)=\sum_{j=1}^{m} a_{j} \otimes f_{i, j}^{\prime}
$$

where $f_{i, j}, f_{i, j}^{\prime} \in \operatorname{End}_{K} B$. If $\left\{\xi_{1}, \xi_{2}, \ldots, \xi_{m}\right\}$ denotes the dual basis of $A^{*}$, then $\left(\xi_{j} \otimes 1\right) f\left(a_{i}\right)=f_{i, j}$ and therefore, by assumption, $\operatorname{tr}_{B} f_{i, j}=\operatorname{tr}_{B} f_{i, j}^{\prime}$ for all $i, j$. Finally observe that $A \otimes B=\oplus \sum_{i} a_{i} \otimes B$ and that

$$
g\left(a_{i} \otimes b\right)=\sum_{j=1}^{m} a_{j} \otimes f_{i, j}(b) .
$$

Thus the subspace $a_{i} \otimes B$ contributes $\operatorname{tr}_{B} f_{i, i}$ to the trace of $g$ and therefore

$$
\operatorname{tr}_{A \otimes B} g=\sum_{i=1}^{m} \operatorname{tr}_{B} f_{i, i}=\sum_{i=1}^{m} \operatorname{tr}_{B} f_{i, i}^{\prime}=\operatorname{tr}_{A \otimes B} g^{\prime}
$$

as required.

We recall from [S] that the subspaces of $H$ given by

$$
\int_{H}^{\ell}=\{x \in H \mid h x=\epsilon(h) x \text { for all } h \in H\}
$$

and

$$
\int_{H}^{r}=\{x \in H \mid x h=\epsilon(h) x \text { for all } h \in H\}
$$

are both one dimensional and that their elements are called the left and right integrals of $H$, respectively. Furthermore, a generaiization of Maschke's theorem asserts that $H$ is semisimple precisely when $H$ has a nonzero idempotent integral. In this case, the spaces of left and right integrals coincide and the 
nonzero idempotent integral is necessarily unique. Since the counit of the dual $H^{*}$ is evaluation at $1_{H}$, we see that

$$
\int_{H^{*}}^{\ell}=\left\{\xi \in H^{*} \mid \mu * \xi=\mu(1) \xi \text { for all } \mu \in H^{*}\right\}
$$

and

$$
\int_{H^{*}}^{r}=\left\{\xi \in H^{*} \mid \xi * \mu=\mu(1) \xi \text { for all } \mu \in H^{*}\right\} .
$$

Now let us define the functionals $\lambda, \lambda^{\prime} \in H^{*}$ by

$$
\lambda(a)=\operatorname{tr}_{H}\left(a_{\mathscr{L}} S^{2}\right) \quad \text { for all } a \in H
$$

and

$$
\lambda^{\prime}(a)=\operatorname{tr}_{H}\left(a_{\mathscr{L}} S^{-2}\right) \quad \text { for all } a \in H
$$

Then we have

Lemma 3. With the above notation, $\lambda$ is a left integral of $H^{*}$ and $\lambda^{\prime}$ is a right integral.

Proof. We begin with $\lambda$. Let $V$ be a left $H$-module, and define the linear transformation $T_{1}: H \otimes V \rightarrow H \otimes V$ by

$$
T_{1}: h \otimes v \mapsto \sum_{(h)} h_{2} \otimes h_{1} v .
$$

Observe that $T_{1}$ is a vector space isomorphism with inverse given by

$$
T_{1}^{-1}: h \otimes v \mapsto \sum_{(h)} h_{2} \otimes S^{-1}\left(h_{1}\right) v .
$$

Now let $a \in H$ and let $L: V \rightarrow V$ be a linear transformation. Then

$$
\lambda(a) \operatorname{tr}_{V} L=\operatorname{tr}_{H}\left(a_{\mathscr{L}} S^{2}\right) \operatorname{tr}_{V} L=\operatorname{tr}_{H \otimes V}\left(a_{\mathscr{L}} S^{2} \otimes L\right)=\operatorname{tr}_{H \otimes V} T_{1}^{-1}\left(a_{\mathscr{L}} S^{2} \otimes L\right) T_{1}
$$

and

$$
T_{1}^{-1}\left(a_{\mathscr{L}} S^{2} \otimes L\right) T_{1}: h \otimes v \mapsto \sum_{(a)(h)} a_{2} S^{2}\left(h_{3}\right) \otimes S\left(h_{2}\right) S^{-1}\left(a_{1}\right) L h_{1} v .
$$

At this point, the preceding lemma comes into play. Specifically, for fixed $a \in H$, we have the linear maps $f, f^{\prime}: H \rightarrow H \otimes \operatorname{End}_{K} V$ given by

$$
f: h \mapsto \sum_{(a)(h)} a_{2} S^{2}\left(h_{3}\right) \otimes S\left(h_{2}\right) S^{-1}\left(a_{1}\right) L h_{1}
$$

and

$$
f^{\prime}: h \mapsto \sum_{(a)(h)} a_{2} S^{2}\left(h_{3}\right) \otimes S^{-1}\left(a_{1}\right) L h_{1} S\left(h_{2}\right) .
$$

Moreover, since

$$
\operatorname{tr}_{V} \sum_{(k)} S\left(k_{2}\right) S^{-1}(b) L k_{1}=\operatorname{tr}_{V} \sum_{(k)} S^{-1}(b) L k_{1} S\left(k_{2}\right)
$$


for all $b, k \in H$, it is easy to verify that the hypotheses of Lemma 2 are satisfied. Thus, it follows that the trace of $T_{1}^{-1}\left(a_{\mathscr{L}} S^{2} \otimes L\right) T_{1}$ is equal to the trace of the linear operator on $H \otimes V$ given by

$$
\begin{aligned}
h \otimes v & \mapsto \sum_{(a)(h)} a_{2} S^{2}\left(h_{3}\right) \otimes S^{-1}\left(a_{1}\right) L h_{1} S\left(h_{2}\right) v \\
& =\sum_{(a)} a_{2} S^{2}(h) \otimes S^{-1}\left(a_{1}\right) L v .
\end{aligned}
$$

As a consequence,

$$
\begin{aligned}
\lambda(a) \operatorname{tr}_{V} L & =\sum_{(a)} \operatorname{tr}_{H}\left(\left(a_{2}\right)_{\mathscr{L}} S^{2}\right) \operatorname{tr}_{V}\left(S^{-1}\left(a_{1}\right) L\right) \\
& =\sum_{(a)} \lambda\left(a_{2}\right) \operatorname{tr}_{V}\left(S^{-1}\left(a_{1}\right) L\right) .
\end{aligned}
$$

Finally, let $V=H$, let $\xi \in H^{*}$, and define $L: H \rightarrow H$ by

$$
L: x \mapsto \xi(S(x)) 1_{H} \quad \text { for all } x \in H .
$$

Then $\operatorname{tr}_{V} L=\xi(1)$ and

$$
\operatorname{tr}_{V}\left(S^{-1}\left(a_{1}\right) L\right)=\operatorname{tr}_{V}\left(L S^{-1}\left(a_{1}\right)\right)=\xi\left(a_{1}\right)
$$

so we obtain

$$
\lambda(a) \xi(1)=\sum_{(a)} \lambda\left(a_{2}\right) \xi\left(a_{1}\right)=(\xi * \lambda)(a) .
$$

Since this is true for all $a \in H$, we conclude that $\xi * \lambda=\xi(1) \lambda$ for all $\xi \in H^{*}$ and therefore $\lambda$ is indeed a left integral of $H^{*}$.

The proof for $\lambda^{\prime}$ is similar. Again let $V$ be a left $H$-module, but this time define $T_{2}: H \otimes V \rightarrow H \otimes V$ by

$$
T_{2}: h \otimes v \mapsto \sum_{(h)} h_{1} \otimes h_{2} v
$$

Then $T_{2}$ is an invertible linear transformation with inverse given by

$$
T_{2}^{-1}: h \otimes v \mapsto \sum_{(h)} h_{1} \otimes S\left(h_{2}\right) v .
$$

Choose $a \in H$, and let $L: V \rightarrow V$ be an arbitrary linear transformation. Then

$$
\begin{aligned}
\lambda^{\prime}(a) \operatorname{tr}_{V} L & =\operatorname{tr}_{H}\left(a_{\mathscr{L}} S^{-2}\right) \operatorname{tr}_{V} L \\
& =\operatorname{tr}_{H \otimes V}\left(a_{\mathscr{L}} S^{-2} \otimes L\right)=\operatorname{tr}_{H \otimes V} T_{2}^{-1}\left(a_{\mathscr{L}} S^{-2} \otimes L\right) T_{2}
\end{aligned}
$$

and

$$
T_{2}^{-1}\left(a_{\mathscr{L}} S^{-2} \otimes L\right) T_{2}: h \otimes v \mapsto \sum_{(a)(h)} a_{1} S^{-2}\left(h_{1}\right) \otimes S^{-1}\left(h_{2}\right) S\left(a_{2}\right) L h_{3} v .
$$

Once again, we apply the previous lemma. Here, for fixed $a \in H$, we have the linear maps $\bar{f}, \bar{f}^{\prime}: H \rightarrow H \otimes \operatorname{End}_{K} V$ given by

$$
\bar{f}: h \mapsto \sum_{(a)(h)} a_{1} S^{-2}\left(h_{1}\right) \otimes S^{-1}\left(h_{2}\right) S\left(a_{2}\right) L h_{3}
$$


and

$$
\bar{f}^{\prime}: h \mapsto \sum_{(a)(h)} a_{1} S^{-2}\left(h_{1}\right) \otimes S\left(a_{2}\right) L h_{3} S^{-1}\left(h_{2}\right)
$$

Moreover,

$$
\operatorname{tr}_{V} \sum_{(k)} S^{-1}\left(k_{1}\right) S(b) L k_{2}=\operatorname{tr}_{V} \sum_{(k)} S(b) L k_{2} S^{-1}\left(k_{1}\right)
$$

for all elements $b, k \in H$, so the assumptions of Lemma 2 are certainly satisfied. Thus it follows that the trace of $T_{2}^{-1}\left(a_{\mathscr{L}} S^{-2} \otimes L\right) T_{2}$ is equal to the trace of the linear operator on $H \otimes V$ given by

$$
\begin{aligned}
h \otimes v & \mapsto \sum_{(a)(h)} a_{1} S^{-2}\left(h_{1}\right) \otimes S\left(a_{2}\right) L h_{3} S^{-1}\left(h_{2}\right) v \\
& =\sum_{(a)} a_{1} S^{-2}(h) \otimes S\left(a_{2}\right) L v .
\end{aligned}
$$

In other words,

$$
\begin{aligned}
\lambda^{\prime}(a) \operatorname{tr}_{V} L & =\sum_{(a)} \operatorname{tr}_{H}\left(\left(a_{1}\right)_{\mathscr{L}} S^{-2}\right) \operatorname{tr}_{V}\left(S\left(a_{2}\right) L\right) \\
& =\sum_{(a)} \lambda^{\prime}\left(a_{1}\right) \operatorname{tr}_{V}\left(S\left(a_{2}\right) L\right) .
\end{aligned}
$$

Finally, suppose $V=H$, choose $\xi \in H^{*}$, and define $L: H \rightarrow H$ by

$$
L: x \mapsto \xi\left(S^{-1}(x)\right) 1_{H} \quad \text { for all } x \in H .
$$

Then $\operatorname{tr}_{V} L=\xi(1)$ and

$$
\operatorname{tr}_{V}\left(S\left(a_{2}\right) L\right)=\operatorname{tr}_{V}\left(L S\left(a_{2}\right)\right)=\xi\left(a_{2}\right)
$$

so we obtain

$$
\lambda^{\prime}(a) \xi(1)=\sum_{(a)} \lambda^{\prime}\left(a_{1}\right) \xi\left(a_{2}\right)=\left(\lambda^{\prime} * \xi\right)(a) .
$$

Since this holds for all $a \in H$, we conclude that $\lambda^{\prime} * \xi=\xi(1) \lambda^{\prime}$ for all $\xi \in H^{*}$ and therefore $\lambda^{\prime}$ is a right integral of $H^{*}$.

With this result in hand, we can proceed as in [L] and [LR2], except that we take an algebra rather than a coalgebra point of view. To start with, recall from [S] that a nonzero left or right integral $\beta$ of $H^{*}$ determines an associative nondegenerate bilinear form $\mathscr{B}: H \times H \rightarrow K$ given by $\mathscr{B}(a, b)=\beta(a b)$ for all $a, b \in H$. In particular, the nondegeneracy implies that $H$ is a Frobenius algebra and that $\beta$ cannot vanish on any nonzero ideal of $H$.

Proposition 4. If $H$ is a semisimple Hopf algebra, then:

(i) $\lambda$ is a nonzero left integral of $H^{*}$ and $\lambda^{\prime}$ is a nonzero right integral.

(ii) $H^{*}$ is semisimple if and only if $\operatorname{tr}_{H}\left(S^{2}\right) \neq 0$.

(iii) $S^{2}$ stabilizes each ideal of $H$.

Proof. (i) If $e$ is the nonzero idempotent integral of $H$, then $S^{2}(e)=e$ and $e$ has rank 1. Thus $\lambda(e)=1=\lambda^{\prime}(e)$ and Lemma 3 yields the result.

(ii) If $\lambda(1)=\operatorname{tr}_{H}\left(S^{2}\right) \neq 0$, then $\lambda(1)^{-1} \lambda$ is a nonzero idempotent integral of $H^{*}$ and therefore $H^{*}$ is semisimple. Conversely, if $H^{*}$ is semisimple, then 
since $\lambda$ is nonzero by (i), some scalar multiple of $\lambda$ is a nonzero idempotent integral. In particular, $0 \neq \lambda * \lambda=\lambda(1) \lambda$ and therefore $\operatorname{tr}_{H}\left(S^{2}\right)=\lambda(1) \neq 0$.

(iii) Let $I$ be a minimal ideal of $H$, and note that $S^{2}(I)=J$ is also a minimal ideal. If $J \neq I$, then $I J=0$ and hence

$$
\left(I_{\mathscr{L}} S^{2}\right)^{2}: H \mapsto I S^{2}(I H)=I J=0 .
$$

In particular, each linear transformation in $I_{\mathscr{L}} S^{2}$ is nilpotent and has trace 0 , so $\lambda(I)=\operatorname{tr}_{H}\left(I_{\mathscr{L}} S^{2}\right)=0$, a contradiction. We conclude therefore that each minimal ideal of $H$ is $S^{2}$-stable and, since $H$ is semisimple, the proposition is proved.

We remark that the converse to (i) above is also true. Specifically, if $\lambda$ or $\lambda^{\prime}$ is nonzero, then $H$ is necessarily semisimple. To see this, suppose that $\lambda \neq 0$ and let $N$ be the radical of $H$. Then $N$ is an $S^{2}$-stable nilpotent ideal of $H$, so $N_{\mathscr{L}} S^{2}$ consists of nilpotent operators on $H$ and $\lambda(N)=\operatorname{tr}_{H}\left(N_{\mathscr{L}} S^{2}\right)=0$. But $\lambda$ is a nonzero left integral of $H^{*}$ by Lemma 3 , so $\lambda$ cannot vanish on a nonzero ideal of $H$ and hence $N=0$, as required.

The proof of the next result requires a small amount of character theory.

Lemma 5. If $H$ is semisimple, then there exists a unique group-like element $g \in H$ such that:

(i) $\lambda * \xi=\xi(g) \lambda$ for all $\xi \in H^{*}$.

(ii) $\lambda(a g)=\lambda^{\prime}(a)$ for all $a \in H$.

(iii) $S^{4}$ is the inner automorphism of $H$ induced by $g$, and $S$ has finite multiplicative order.

Furthermore, if char $K=0$, then $\operatorname{tr}_{H}\left(S^{2}\right)$ is real and positive.

Proof. (i) We know that $\int_{H^{*}}^{\ell}$ is the one-dimensional $K$-space spanned by $\lambda$, and it is easy to see that $\lambda * \xi$ is a left integral for any $\xi \in H^{*}$. Thus, since $H^{* *}=$ $H$, there exists a unique element $g \in H$ with $\lambda * \xi=\xi(g) \lambda$ for all $\xi \in H^{*}$. Moreover, the map $g: H^{*} \rightarrow K$ given by $\xi \mapsto \xi(g)$ is clearly multiplicative, and from this and the definition of multiplication in $H^{*}$, it follows that $g$ is a group-like element of $H$. In particular, $g$ is invertible in $H$ and in fact $g$ has finite multiplicative order.

(ii) We now show that the functional $\lambda^{g} \in H^{*}$ given by $\lambda^{g}(a)=\lambda(a g)$ for all $a \in H$ is a right integral of $H^{*}$. To this end, observe that since $g$ is invertible in $H$, any element of $H^{*}$ is of the form $\xi^{g}: a \mapsto \xi(a g)$ for some $\xi \in H^{*}$. Furthermore, since $g$ is a group-like element, we have

$$
\begin{aligned}
\left(\lambda^{g} * \xi^{g}\right)(a) & =\sum_{(a)} \lambda^{g}\left(a_{1}\right) \xi^{g}\left(a_{2}\right)=\sum_{(a)} \lambda\left(a_{1} g\right) \xi\left(a_{2} g\right) \\
& =\sum_{(a g)} \lambda\left((a g)_{1}\right) \xi\left((a g)_{2}\right)=(\lambda * \xi)(a g)=(\lambda * \xi)^{g}(a)
\end{aligned}
$$

for all $a \in H$. Thus, by (i) above,

$$
\lambda^{g} * \xi^{g}=(\lambda * \xi)^{g}=(\xi(g) \lambda)^{g}=\xi(g) \lambda^{g}=\xi^{g}(1) \lambda^{g}
$$

and $\lambda^{g}$ is indeed a right integral of $H^{*}$. In particular, $\lambda^{g}$ is a scalar multiple of $\lambda^{\prime}$. But if $e$ denotes the nonzero idempotent integral of $H$, then since $e g=\epsilon(g) e=e$, we have

$$
\lambda^{g}(e)=\lambda(e g)=\lambda(e)=1=\lambda^{\prime}(e)
$$

and therefore $\lambda^{g}=\lambda^{\prime}$. 
(iii) Let $\bar{K}$ denote the algebraic closure of $K$, and let $\bar{H}=H \otimes \bar{K} \supseteq H$. Since the nonzero idempotent integral of $H$ is clearly an idempotent integral of $\bar{H}$, it follows that $\bar{H}$ is also semisimple. Furthermore, since $\bar{H}^{*}=H^{*} \otimes \bar{K} \supseteq H^{*}$, we see that the corresponding elements $\bar{\lambda}$ and $\bar{\lambda}^{\prime}$ of $\bar{H}^{*}$ are the natural extensions of $\lambda$ and $\lambda^{\prime}$, respectively. With these observations, it clearly suffices to work in $\bar{H}$, or equivalently, we can now assume that $K=\bar{K}$ is algebraically closed.

Write $H=\bigoplus \sum_{i=1}^{q} I_{i}$ as the direct sum of its minimal two-sided ideals. Since $K$ is algebraically closed, each such minimal ideal is isomorphic to a full matrix ring over $K$; say $I_{i}=\mathrm{M}_{d_{i}}(K)$ and let $\chi_{i}: \mathrm{M}_{d_{i}}(K) \rightarrow K$ denote the usual matrix trace. If $a, b \in I_{i}$, then the map $\theta_{a, b}: x \mapsto a x b$ defines a linear operator on $I_{i}$ and, as is well known, $\operatorname{tr}_{I_{i}} \theta_{a, b}=\chi_{i}(a) \chi_{i}(b)$.

By Proposition 4(iii), $I_{i}$ is $S^{2}$-stable and therefore, by the Noether-Skolem Theorem, there exists a unit $c_{i}$ of $I_{i}$ such that $S^{2}(x)=c_{i}^{-1} x c_{i}$ for all $x \in I_{i}$. Here, of course, $c_{i}^{-1}$ is the "local inverse" of $c_{i}$ in $I_{i}$. Let $y \in I_{i}$. Since the linear transformation $y_{\mathscr{L}} S^{2}: H \rightarrow H$ annihilates all the minimal ideals $I_{j}$ of $H$ with $j \neq i$, we have

$$
\lambda(y)=\operatorname{tr}_{H}\left(y_{\mathscr{L}} S^{2}\right)=\operatorname{tr}_{I_{i}}\left(y_{\mathscr{L}} S^{2}\right) .
$$

Hence, since

$$
y_{\mathscr{L}} S^{2}: x \mapsto\left(y c_{i}^{-1}\right) x c_{i} \quad \text { for all } x \in I_{i},
$$

it follows that

$$
\lambda(y)=\operatorname{tr}_{I_{i}}\left(y_{\mathscr{L}} S^{2}\right)=\chi_{i}\left(y c_{i}^{-1}\right) \chi_{i}\left(c_{i}\right) .
$$

In a similar manner, we obtain

$$
\lambda^{\prime}(y)=\operatorname{tr}_{I_{i}}\left(y_{\mathscr{L}} S^{-2}\right)=\chi_{i}\left(y c_{i}\right) \chi_{i}\left(c_{i}^{-1}\right)
$$

and, as we observed earlier, neither $\lambda$ nor $\lambda^{\prime}$ can vanish on $I_{i}$. As a consequence, $\chi_{i}\left(c_{i}\right)$ and $\chi_{i}\left(c_{i}^{-1}\right)$ are both nonzero scalars.

Now, by (ii) above, $\lambda^{\prime}(y)=\lambda(y g)$ and therefore

$$
\chi_{i}\left(y c_{i}\right) \chi_{i}\left(c_{i}^{-1}\right)=\lambda^{\prime}(y)=\lambda(y g)=\chi_{i}\left(y g_{i} c_{i}^{-1}\right) \chi_{i}\left(c_{i}\right)
$$

where $g_{i}$ denotes the projection of $g$ into $I_{i}$. Since the above holds for all $y \in I_{i}$, we see that $\chi_{i}$ vanishes on the left ideal of $I_{i}$ generated by the element

$$
c_{i} \chi_{i}\left(c_{i}^{-1}\right)-g_{i} c_{i}^{-1} \chi_{i}\left(c_{i}\right)
$$

and so this element must be zero. In particular, we have $c_{i}^{2}=g_{i} \chi_{i}\left(c_{i}\right) / \chi_{i}\left(c_{i}^{-1}\right)$ and conclude that

$$
S^{4}(x)=c_{i}^{-2} x c_{i}^{2}=g_{i}^{-1} x g_{i} \quad \text { for all } x \in I_{i} .
$$

It is now clear that $S^{4}$ is the inner automorphism of $H$ induced by $g$, and part (iii) follows since $g$ has finite multiplicative order.

Finally suppose char $K=0$ and say $S^{2 w}=\mathrm{id}_{H}$. Then $c_{i}^{w \prime}$ is a nonzero scalar matrix in $\mathbf{M}_{d_{i}}(K)$; and since $K$ is algebraically closed, we can multiply $c_{i}$ by a suitable scalar to assume that $c_{i}^{u \prime}=1_{I_{i}}$. Of course, this implies that the eigenvalues of $c_{i}^{-1}$ are the complex conjugates of the eigenvalues of $c_{i}$ and 
consequently $\chi_{i}\left(c_{i}^{-1}\right)$ is the complex conjugate of $\chi_{i}\left(c_{i}\right)$. Thus since $\operatorname{tr}_{H}\left(S^{2}\right)=$ $\lambda(1)$ and each $\chi_{i}\left(c_{i}\right)$ is nonzero, we have

$$
\operatorname{tr}_{H}\left(S^{2}\right)=\sum_{i=1}^{q} \chi_{i}\left(c_{i}^{-1}\right) \chi_{i}\left(c_{i}\right)=\sum_{i=1}^{q}\left|\chi_{i}\left(c_{i}\right)\right|^{2}>0
$$

and the lemma is proved.

Recall that a finite-dimensional Hopf algebra is said to be unimodular if its left and right integral subspaces coincide. As we observed earlier, any semisimple Hopf algebra is necessarily unimodular.

Corollary 6. Let $H$ be semisimple.

(i) If $H^{*}$ is unimodular, then $S^{4}=\mathrm{id}_{H}$.

(ii) If char $K=0$, then $H^{*}$ is semisimple.

Proof. (i) Since $H^{*}$ is unimodular, $\lambda$ is a right integral for $H^{*}$ and therefore

$$
\xi(1) \lambda=\lambda * \xi=\xi(g) \lambda \quad \text { for all } \xi \in H^{*}
$$

where $g$ is the group-like element of $H$ given by part (i) of the previous lemma. It is now clear that $g=1$ and hence $S^{4}=\mathrm{id}_{H}$ by Lemma 5 (iii).

(ii) Since char $K=0$, the previous lemma implies that $\operatorname{tr}_{H}\left(S^{2}\right) \neq 0$ and it follows from Proposition 4(ii) that $H^{*}$ is semisimple.

We can now obtain the main result of [LR2] using the key argument of that paper.

Theorem 7. Let $H$ be a finite-dimensional Hopf algebra over the field $K$, and assume that both $H$ and its dual are semisimple. Then $\operatorname{dim}_{K} H \neq 0$ in $K$. Furthermore, if either char $K=0$ or char $K=p>\left(\operatorname{dim}_{K} H\right)^{2}$, then $H$ is involutory.

Proof. It suffices to assume that $m=\operatorname{dim}_{K} H>1$. We continue with all the previous notation and, in addition, let $t \in H=H^{* *}$ correspond to the trace map on $H^{*}$. In other words, the trace element $t \in H$ is defined by

$$
\xi(t)=\operatorname{tr}_{H^{*}} \xi_{\mathscr{L}} \quad \text { for all } \xi \in H^{*} .
$$

Clearly

$$
\epsilon(t)=1_{H^{*}}(t)=\operatorname{dim}_{K} H^{*}=\operatorname{dim}_{K} H
$$

and, by applying Lemma 1 to $H^{*}$, we see that $t^{2}=\left(\operatorname{dim}_{K} H\right) t$ and that $S^{2}$ and $t_{\mathscr{L}}$ commute as operators on $H$.

Now, by assumption, $H^{*}$ is semisimple, so $\lambda(1)^{-1} \lambda$ is a nonzero idempotent of rank 1 by Proposition 4 and therefore

$$
\operatorname{tr}_{H} S^{2}=\lambda(1)=\operatorname{tr}_{H^{*}} \lambda_{\mathscr{L}} \text {. }
$$

Furthermore, by definitions of $t$ and $\lambda$, we have

$$
\operatorname{tr}_{H^{*}} \lambda_{\mathscr{L}}=\lambda(t)=\operatorname{tr}_{H}\left(t_{\mathscr{L}} S^{2}\right) .
$$

Thus

$$
\operatorname{tr}_{H}\left(t_{\mathscr{L}} S^{2}\right)=\operatorname{tr}_{H} S^{2}=\lambda(1) \neq 0
$$


and, in particular, $t_{\mathscr{L}} S^{2}$ is not nilpotent. Since $S^{2}$ and $t_{\mathscr{L}}$ commute and since $t^{2}=\left(\operatorname{dim}_{K} H\right) t$, it therefore follows that $\operatorname{dim}_{K} H$ is not zero in $K$. This also implies that $f=\left(\operatorname{dim}_{K} H\right)^{-1} t$ is a nonzero idempotent of $H$. Moreover, since $t_{\mathscr{L}} S^{2}=S^{2} t_{\mathscr{L}}$ annihilates $(1-f) H$ and acts like $\left(\operatorname{dim}_{K} H\right) S^{2}$ on $f H$, we have

$$
\operatorname{tr}_{f H} S^{2}+\operatorname{tr}_{(1-f) H} S^{2}=\operatorname{tr}_{H} S^{2}=\operatorname{tr}_{H}\left(t_{\mathscr{L}} S^{2}\right)=\left(\operatorname{dim}_{K} H\right) \operatorname{tr}_{f H} S^{2}
$$

and obtain the trace equation

$$
\operatorname{tr}_{(1-f) H} S^{2}=\left(\operatorname{dim}_{K} H-1\right) \operatorname{tr}_{f H} S^{2} .
$$

By the preceding corollary, $\left(S^{2}\right)^{2}=\mathrm{id}_{H}$ and, by hypothesis, char $K \neq 2$. Thus $S^{2}$ is diagonalizable in its action on $H$ and in fact $H=E_{1} \oplus E_{-1}$ where

$$
E_{i}=\left\{h \in H \mid S^{2}(h)=i h\right\}
$$

for $i= \pm 1$. With this, we can define the integers $n, n^{\prime}$ by

$$
n=\operatorname{dim}_{K}\left(f H \cap E_{1}\right)-\operatorname{dim}_{K}\left(f H \cap E_{-1}\right)
$$

and

$$
n^{\prime}=\operatorname{dim}_{K}\left((1-f) H \cap E_{1}\right)-\operatorname{dim}_{K}\left((1-f) H \cap E_{-1}\right) .
$$

Then clearly, $|n| \leq \operatorname{dim}_{K} f H,\left|n^{\prime}\right| \leq \operatorname{dim}_{K}(1-f) H$, and $|n|+\left|n^{\prime}\right| \leq m=$ $\operatorname{dim}_{K} H$. Furthermore,

$$
\operatorname{tr}_{f H} S^{2}={ }_{K} n \quad \text { and } \quad \operatorname{tr}_{(1-f) H} S^{2}={ }_{K} n^{\prime}
$$

where we temporarily use $=_{K}$ to indicate equality in the field $K$. Thus the trace equation becomes

$$
n^{\prime}={ }_{K}(m-1) n \text {. }
$$

Furthermore, notice that $\left|(m-1) n-n^{\prime}\right| \leq m^{2}$ and that, by hypothesis, this is less than char $K$ if char $K=p>0$. Thus, in all cases, we obtain

$$
n^{\prime}=(m-1) n
$$

where this is an equation in the integers.

Finally, if $n=0$, then $n^{\prime}=(m-1) n=0$ and $\operatorname{tr}_{K} S^{2}={ }_{K} n+n^{\prime}=0$, a contradiction. Thus, $|n| \geq 1$, and from $(m-1) \geq\left|n^{\prime}\right|=(m-1)|n|$ it follows that $n= \pm 1$ and $n^{\prime}= \pm(m-1)$ with the same sign. In particular, $|n|+\left|n^{\prime}\right|=m$, and this and the common sign imply that $H=E_{1}$ or $H=E_{-1}$. But $1_{H} \in E_{1}$, so $E_{1} \neq 0$; therefore, $H=E_{1}$ and $S^{2}=\mathrm{id}_{H}$, as required.

A generalization and other applications of the key trace formula $\operatorname{tr}_{H}\left(t_{\mathscr{L}} S^{2}\right)=$ $\left(\operatorname{dim}_{K} H\right) \operatorname{tr}_{f H} S^{2}$ can be found in [R1]. Related formulas and their applications are contained in [R2]. Kaplansky's conjecture in characteristic 0 is now an immediate consequence of Corollary 6(ii) and Theorem 7. Specifically, we have

Corollary 8. A semisimple Hopf algebra in characteristic 0 is involutory.

We close this paper with another relationship between this problem and the linear transformation $T$ of the introduction. To start with, recall that the trace element $\tau \in H^{*}$ is defined by

$$
\tau(a)=\operatorname{tr}_{H}\left(a_{\mathscr{L}}\right) \quad \text { for all } a \in H
$$


and that $\lambda \in H^{*}$ is given by

$$
\lambda(a)=\operatorname{tr}_{H}\left(a_{\mathscr{L}} S^{2}\right) \quad \text { for all } a \in H .
$$

Thus if $S^{2}=\mathrm{id}_{H}$, then certainly $\tau=\lambda$. Conversely, if $\tau=\lambda$ and if $H$ is semisimple, then the character argument of Lemma 5(iii) easily proves that $S^{2}=\mathrm{id}_{H}$. In particular, an alternate approach to Theorem $7 \mathrm{might}$ be to somehow show directly that $\tau=\lambda$. In fact, since $\tau(1)^{-1} \tau$ is an idempotent of $H^{*}$ with $\tau(1)^{-1} \tau * \lambda(1)^{-1} \lambda=\lambda(1)^{-1} \lambda$, the equality could be achieved by showing that $\tau(1)^{-1} \tau$ has rank 1 or equivalently, when char $K$ is big enough, that $\operatorname{tr}_{H^{*}} \tau_{\mathscr{L}}=\tau(1)$. We consider this trace equation from a dual point of view. Specifically, let $t$ be the trace element of $H$ defined by

$$
\xi(t)=\operatorname{tr}_{H^{*}} \xi_{\mathscr{L}} \quad \text { for all } \xi \in H^{*} .
$$

Then we have

Lemma 9. Let $\Delta: H \rightarrow H \otimes H$ denote the comultiplication of the Hopf algebra $H$, and let $t$ be its trace element.

(i) If $\left\{x_{1}, x_{2}, \ldots, x_{m}\right\}$ is a basis for $H$ and if $x_{i, j}$ and $x_{i, j}^{\prime}$ are the elements of $H$ uniquely determined by the equations

$$
\Delta\left(x_{i}\right)=\sum_{j=1}^{m} x_{j} \otimes x_{i, j}=\sum_{j=1}^{m} x_{i, j}^{\prime} \otimes x_{j}
$$

for $i=1,2, \ldots, m$, then

$$
t=\sum_{i=1}^{m} x_{i, i}=\sum_{i=1}^{m} x_{i, i}^{\prime} .
$$

(ii) If $T, T^{\prime}: H \otimes H \rightarrow H \otimes H$ are the linear operators given by

$$
T: h \otimes v \mapsto \sum_{(h)} h_{1} \otimes h_{2} v \quad \text { and } \quad T^{\prime}: h \otimes v \mapsto \sum_{(h)} h_{2} \otimes h_{1} v
$$

for all $h, v \in H$, then

$$
\operatorname{tr}_{H} t_{\mathscr{L}}=\operatorname{tr}_{H \otimes H} T=\operatorname{tr}_{H \otimes H} T^{\prime}
$$

Proof. (i) Let $\left\{\xi_{1}, \xi_{2}, \ldots, \xi_{m}\right\}$ be the dual basis for $H^{*}$ corresponding to the given basis $\left\{x_{1}, x_{2}, \ldots, x_{m}\right\}$ for $H$. Then for all $i, j, k$ we have

$$
\left(\xi_{i} * \xi_{j}\right)\left(x_{k}\right)=\sum_{q=1}^{m} \xi_{i}\left(x_{k, q}^{\prime}\right) \xi_{j}\left(x_{q}\right)=\xi_{i}\left(x_{k, j}^{\prime}\right)
$$

and hence

$$
\xi_{i} * \xi_{j}=\sum_{k=1}^{m} \xi_{i}\left(x_{k, j}^{\prime}\right) \xi_{k} .
$$

By definition of $t$, it follows that

$$
\xi_{i}(t)=\operatorname{tr}_{H^{*}}\left(\xi_{i}\right)_{\mathscr{L}}=\sum_{i=1}^{m} \xi_{i}\left(x_{j, j}^{\prime}\right)=\xi_{i}\left(\sum_{j=1}^{m} x_{j, j}^{\prime}\right) ;
$$

and since this holds for all $i$, we conclude that $t=\sum_{j=1}^{m} x_{j, j}^{\prime}$. 
In a similar manner, using the defining equations for the $x_{i, j}$, we can compute the trace of right multiplication by $\xi_{j}$. But $H^{*}$ is a Frobenius algebra, so left and right multiplication by $\xi_{j}$ have the same trace and with this we obtain $t=\sum_{i=1}^{m} x_{i, i}$.

(ii) Notice that $H \otimes H=\oplus \sum_{i=1}^{m} x_{i} \otimes H$ and that

$$
T: x_{i} \otimes v \mapsto \sum_{j=1}^{m} x_{j} \otimes x_{i, j} v \quad \text { for all } v \in H .
$$

Thus the subspace $x_{i} \otimes H$ contributes $\operatorname{tr}_{H}\left(x_{i, i}\right)_{\mathscr{L}}$ to the trace of $T$ and therefore

$$
\operatorname{tr}_{H \otimes H} T=\sum_{i=1}^{m} \operatorname{tr}_{H}\left(x_{i, i}\right)_{\mathscr{L}}=\operatorname{tr}_{H} t_{\mathscr{L}}
$$

by (i) above. Similarly

$$
\operatorname{tr}_{H \otimes H} T^{\prime}=\sum_{i=1}^{m} \operatorname{tr}_{H}\left(x_{i, i}^{\prime}\right) \mathscr{L}=\operatorname{tr}_{H} t_{\mathscr{L}},
$$

and the result follows.

Thus a direct computation of $\operatorname{tr}_{H \otimes H} T$, perhaps under the assumption that $H$ or $H^{*}$ is semisimple, could prove to have interesting consequences. We of course expect this trace to equal $\operatorname{dim}_{K} H$.

\section{REFERENCES}

[K] I. Kaplansky, Bialgebras, University of Chicago Lecture Notes in Math., Chicago, 1975.

[L] R. G. Larson, Characters of Hopf algebra, J. Algebra 17 (1971), 352-368.

[LR1] R. G. Larson and D. E. Radford, Finite dimensional cosemisimple Hopf algebras in characteristic 0 are semisimple, J. Algebra 117 (1988), 267-289.

[LR2] __, Semisimple cosemisimple Hopf algebras, Amer. J. Math. 109 (1987), 187-195.

[N] W. D. Nichols, Cosemisimple Hopf algebras, Advances in Hopf Algebras, Lecture Notes in Pure and Appl. Math., vol. 158, Dekker, New York, 1994, pp. 135-151.

[R1] D. E. Radford, The group of automorphisms of a semisimple Hopf algebra over a field of characteristic 0 is finite, Amer. J. Math. 112 (1990), 331-357.

[R2] _ The trace function and Hopf algebras, J. Algebra 163 (1994), 583-622.

[S] M. E. Sweedler, Hopf algebras, Benjamin, New York, 1969. 53706

Department of Mathematics, University of Wisconsin-Madison, Madison, Wisconsin E-mail address: passman@math.wisc.edu

Department of Mathematics, Syracuse University, Syracuse, New York 13244

E-mail address: dpquinn@mailbox.syr.edu 